\title{
Pharmacogenomic analyses of sunitinib in patients with pancreatic neuroendocrine tumors
}

\author{
Nicola Fazio ${ }^{1}$, Jean-Francois Martini ${ }^{2}$, Adina E Croitoru ${ }^{3}$, Michael Schenker ${ }^{4}$, Sherry Li ${ }^{2}$, \\ Brad Rosbrook $^{2}$, Kathrine Fernandez ${ }^{5}$, Jiri Tomasek ${ }^{6}$, Espen Thiis-Evensen ${ }^{7}$, Matthew \\ Kulke $^{8}$ \& Eric Raymond*,9 \\ ${ }^{1}$ Division of Gastrointestinal Medical Oncology \& Neuroendocrine Tumors, European Institute of Oncology, IEO, IRCCS, Milan, Italy \\ 2 Pfizer Inc, La Jolla, CA, USA \\ ${ }^{3}$ Department of Medical Oncology, Fundeni Clinical Institute, Bucharest, Romania \\ ${ }^{4}$ Centrul de Oncologie Sf. Nectarie, Oncologie Medicala, Craiova, Romania \\ ${ }^{5}$ Pfizer Inc, Cambridge, MA, USA \\ ${ }^{6}$ Faculty of Medicine, Masaryk Memorial Cancer Institute, Masaryk University, Brno, Czech Republic \\ ${ }^{7}$ Department of Gastroenterology, Oslo University Hospital, Rikshospitalet, Oslo, Norway \\ ${ }^{8}$ Boston University \& Boston Medical Center, Boston, MA, USA \\ ${ }^{9}$ Department of Medical Oncology, Paris Saint-Joseph Hospital Group, Paris, France \\ *Author for correspondence: Tel.: +33 014412 7881; eraymond@hpsj.fr
}

Aim: Evaluate associations between clinical outcomes and SNPs in patients with well-differentiated pancreatic neuroendocrine tumors receiving sunitinib. Patients \& methods: Kaplan-Meier and Cox proportional hazards models were used to analyze the association between SNPs and survival outcomes using data from a sunitinib Phase IV (genotyped, $n=56$ ) study. Fisher's exact test was used to analyze objective response rate and genotype associations. Results: After multiplicity adjustment, progression-free and overall survivals were not significantly correlated with SNPs; however, a higher objective response rate was significantly associated with IL1B rs $16944 \mathrm{G} / \mathrm{A}$ versus $\mathrm{G} / \mathrm{G}$ (46.4 vs $4.5 \% ; \mathrm{p}=0.001)$. Conclusion: IL1B SNPs may predict treatment response in patients with pancreatic neuroendocrine tumors. VEGF pathway SNPs are potentially associated with survival outcomes.

First draft submitted: 17 December 2018; Accepted for publication: 14 March 2019; Published online: 14 May 2019

Keywords: biomarkers $\bullet$ efficacy $\bullet$ pancreatic neuroendocrine tumor $\bullet$ sunitinib $\bullet$ VEGF

Pancreatic neuroendocrine tumors (panNETs) are rare malignancies; however, the incidence of these tumors appears to be increasing [1,2]. A number of options are available for the treatment of unresectable disease, including cytotoxic chemotherapy; somatostatin analogs; peptide receptor radionuclide therapy; and targeted agents, such as the mTOR inhibitor everolimus and the multitarget tyrosine kinase inhibitor (TKI) sunitinib [3]. However, most of these treatments may eventually be associated with either a primary resistance or a progressive loss of antitumor activity. Furthermore, not all therapies are approved by the European Medicines Agency (EMA) or US FDA [3].

Sunitinib is a TKI that inhibits the VEGFR signalling pathway, including VEGFR1-VEGFR3. panNETs are highly vascular tumors, and overexpression of VEGF has been shown to promote the growth of panNETs through increased angiogenesis [4]. Sunitinib is approved in the USA and Europe for the treatment of progressive, well-differentiated panNETs in patients with unresectable locally advanced or metastatic disease [5]. A Phase IV trial (ClinicalTrials.gov, NCT01525550) was conducted to provide additional information in previously treated patients and patients who were treatment-naive. Results in this Phase IV trial with a median progression-free survival (PFS) of 13.2 months, objective response rate (ORR) $24.5 \%$ and median overall survival (OS) of 37.8 months in sunitinib-treated patients with well-differentiated panNETs [6], were consistent with that of the Phase III study [5]. As a consequence of this demonstrated clinical activity, European Society of Medical Oncology (ESMO) clinical practice guidelines recommend sunitinib as a treatment option for patients with unresectable panNETs [7]. Despite publication of a treatment algorithm, the optimal allocation and sequence of treatments, 
particularly among targeted therapies, requires an individualized approach, carefully balancing tolerability and efficacy considerations [8]. Real-world clinical experience with sunitinib in patients with panNETs demonstrates that sunitinib is a safe and effective treatment in patients with well-differentiated tumors [9] as well as heavily pretreated patients [10]. In two open-label extension studies of the sunitinib Phase III trial population, sunitinib was well-tolerated in the longer term [11], was consistent with the safety profile from the original Phase III trial [5] and other indications [12].

Not all patients treated with sunitinib experience benefit, and interpatient variability may be at least in part related with polymorphisms of sunitinib targets and other proteins involved in inflammation and metabolism. Being able to identify those patients most likely to benefit from a certain treatment may both improve the overall effectiveness of treatment and minimize unnecessary treatment-related adverse events. One way to achieve this is by defining predictive biomarkers. SNPs, the most common type of genetic variation, are stable single-base substitutions present in $>1 \%$ of a population [13]. Germline SNPs, that is, those not arising from tumor cells, are attractive biomarker candidates as they are readily accessible via blood samples. As SNPs may alter drug metabolism and drug targets or effectors, there may be direct consequences for dosing, efficacy and safety [14]. Thus, pharmacogenomic evaluations have been developed as a per protocol ancillary analysis to evaluate potential associations between patient genotypes and clinical outcomes. The genes selected for this current study were $A B C B 1, V E G F A, V E G F R 2 / K D R$, VEGFRI and IL1B, based on prior studies showing trends of correlations [15-19]. Therefore, the objectives of these exploratory analyses were to evaluate potential associations between clinical outcomes and SNPs in genes involved in angiogenesis, protein transport or inflammatory response, using a subset of patients from the Phase IV trial of sunitinib treatment in patients with panNETs.

\section{Methods}

Study design \& patients

This was a single-arm, open-label, Phase IV clinical trial of sunitinib in patients with locally advanced unresectable/metastatic, well-differentiated panNETs. The study was conducted in accordance with the protocol, international ethical and clinical practice guidelines, the Declaration of Helsinki, and applicable local regulatory requirements and laws. All patients provided informed consent. The trial is registered on ClinicalTrials.gov (NCT01525550).

Patient eligibility has been reported previously [6]. Briefly, patients were $\geq 18$ years old (in Japan, $\geq 20$ years) with a histologically or cytologically proven diagnosis of well-differentiated panNETs and unresectable or metastatic disease with documented radiologic progression. Prior treatment with TKIs, anti-VEGF, non-VEGF angiogenesis inhibitors or mTOR inhibitors was not permitted [6].

Patients received $37.5 \mathrm{mg}$ sunitinib orally once daily on a continuous daily dosing regimen. Dose modifications were permitted at the investigator's discretion. Patients were treated until death, unacceptable toxicity, withdrawal or the final analysis for the study was performed. Patients with evidence of disease progression could continue treatment if it was judged to have clinical benefit [6].

\section{Molecular biomarker assays}

Anonymized blood samples were prospectively collected from patients in the Phase IV trial who consented to the pharmacogenomics analyses. Samples were genotyped for 12 SNPs previously associated with panNET risk, prognosis or drug effect (Table 1). Samples underwent DNA extraction and DNA amplification using PCR. Commercially available TaqMan ${ }^{\circledR}$ assays (Thermo Fisher Scientific, MA, USA) were used and analyzed on an Applied Biosystems QuantStudio ${ }^{T M}$ 12K Flex Real-Time PCR System (Thermo Fisher Scientific; dba: Life Technologies), and was performed at Pfizer Clinical Pharmacogenomics Laboratory (Pfizer Inc, CT, USA).

\section{Statistical analyses}

The primary end point was investigator-assessed PFS per Response Evaluation Criteria In Solid Tumors (RECIST v1.0). PFS was defined as the time from enrollment to first progression of disease or death (in the absence of documented progressive disease), whichever occurred first. OS and ORR (investigator assessed) were assessed as secondary end points.

The pharmacogenomics population comprised all patients who received at least one dose of study medication (i.e., treatment assignments designated according to actual study treatment received) and who had at least one genotype result. Fisher's exact test was used to compare baseline demographics and characteristics between treatment 


\begin{tabular}{|c|c|c|c|c|}
\hline Official gene symbol & Alternate name & Official SNP \& allele change & mRNA position \& allele change & Protein position and changes \\
\hline$A B C B 1$ & $\begin{array}{l}C L C S ; M D R 1 ; P-G P ; P G Y 1 ; \\
A B C 20 ; C D 243 ; G P 170\end{array}$ & $r s 1128503(\mathrm{C}>\mathrm{T})$ & c. $1236 \mathrm{~T}>$ C, NM_000927.4 & Gly412= \\
\hline$A B C B 1$ & $\begin{array}{l}C L C S ; M D R 1 ; P-G P ; P G Y 1 ; \\
A B C 20 ; C D 243 ; G P 170\end{array}$ & $r s 1045642(C>T)$ & c.3435T>C, NM_000927.4: & Ile1145= \\
\hline VEGFA & VEGF, VPF, MVCD1, MGC70609 & $r s 833061(\mathrm{~T}>\mathrm{C})$ & c. $-958 \mathrm{C}>\mathrm{T}$, NM_001025366.2 & - \\
\hline VEGFA & VEGF, VPF, MVCD1, MGC70609 & $r s 833068(\mathrm{G}>\mathrm{A})$ & $\begin{array}{l}\text { c. } 658+398 G>A \\
\text { NM_001025366.2 }\end{array}$ & - \\
\hline VEGFR2 & $K D R, F L K 1 ; C D 309 ;$ VEGFR & $r s 7692791(\mathrm{~T}>\mathrm{C})$ & c. $798+54 G>$ A, NM_002253.2 & - \\
\hline VEGFR2 & KDR, FLK1; CD309; VEGFR & $r s 1870377(\mathrm{~T}>\mathrm{A})$ & c. $1416 \mathrm{~A}>\mathrm{T}, \mathrm{NM} \_002253.2$ & Exon $11, \mathrm{G} \ln 472 \mathrm{His}$ \\
\hline VEGFR1 & $F L T ; F L T-1 ;$ VEGFR-1 & $r s 9554320(\mathrm{C}>\mathrm{A})$ & c.3387-692T>G, NM_002019.4. & - \\
\hline
\end{tabular}

panNET: Pancreatic neuroendocrine tumor; SNP: Single nucleotide polymorphism.

groups. Two-sided 95\% CIs for allele frequency and genotype frequency were determined by exact method using the F distribution. All SNPs were examined for deviation from Hardy-Weinberg equilibrium as a quality control measure. All p-values were calculated using Pearson's $\chi^{2}$ test based on 10,000 replicates. Linkage disequilibrium analysis was also performed for SNP pairs. $\mathrm{D}^{\prime}, \mathrm{r} 2$ and $\mathrm{p}$-values were calculated for each pair of SNPs within a given gene or on the same chromosome.

In exploratory analyses, associations between SNPs and PFS or OS were assessed using Kaplan-Meier analysis and Cox proportional hazards models. Genotypes were compared within treatment-naive, previously treated and combined groups, and treatment groups were compared within genotypes. Fisher's exact test was used for association between ORR and genotype. All p-values were unadjusted for multiplicity; tests were significant if the p-value was $<0.05$.

To adjust for multiplicity in testing for an association between a genotype and a clinical end point, the Bonferroni multiplicity adjustment method was used. Considering the 12 SNPs in this study, the adjusted significance level was $0.05 / 12=0.0041$; tests were significant if the p-value was $<0.0041$. The data cut-off date for these pharmacogenomic analyses was 19 March 2016, per the primary analyses [6].

\section{Results}

Patients

From a total of 106 patients enrolled in the clinical study, 56 patients ( 25 treatment-naive and 31 previously treated) consented, provided a blood sample and were genotyped. Baseline demographics were generally comparable between groups (Table 2), with the following exceptions: there were more female patients $(56.0 \%)$ in the treatment-naive cohort versus the previously treated cohort (25.8\%). Additionally, there were no Asian patients in the genotyped subpopulation compared with $74.2 \%$ of the nongenotyped subpopulation.

\section{Genotyping}

There were no unexpected findings in allele and genotype frequencies for SNPs (Supplementary Tables 1 \& 2). In general, there were no deviations from Hardy-Weinberg equilibrium; high-linkage disequilibrium was detected between SNPs VEGFA rs2010963, VEGFA rs833068 and VEGFA rs833061 on chromosome 6 (D' = 1.000, r2 ranging from 0.411 to $1.000 ; \mathrm{p}<0.001$ ), between VEGFR1 rs9554320 and VEGFR1 rs9582036 on chromosome $13\left(\mathrm{D}^{\prime}=1.000, \mathrm{r} 2=0.540 ; \mathrm{p}<0.001\right)$, and between SNPs $A B C B 1$ rs $1045642, A B C B 1$ rs 1128503 and $A B C B 1$ rs2032582 on chromosome $7\left(\mathrm{D}^{\prime}>0.9\right.$, r2, ranging from 0.611 to $\left.0.785 ; \mathrm{p}<0.001\right)$, as expected for SNPs located on the same gene. 
Table 2. Patient baseline characteristics.

\begin{tabular}{|c|c|c|c|c|c|c|}
\hline \multirow[t]{2}{*}{ Characteristic } & \multicolumn{3}{|c|}{ All patients } & \multicolumn{3}{|c|}{ Genotyped } \\
\hline & $\begin{array}{l}\text { All genotyped } \\
(n=56)\end{array}$ & $\begin{array}{l}\text { Nongenotyped } \\
(n=50)\end{array}$ & $\begin{array}{l}\text { p-value }{ }^{\dagger} \text { (genotyped } \\
\text { vs nongenotyped) }\end{array}$ & $\begin{array}{l}\text { Treatment-naive } \\
(n=25)\end{array}$ & $\begin{array}{l}\text { Previously treated } \\
(n=31)\end{array}$ & $\begin{array}{l}\mathrm{p} \text {-value }{ }^{\dagger} \text { (treatment } \\
\text { naive vs previously } \\
\text { treated) }\end{array}$ \\
\hline \multicolumn{7}{|l|}{ Age, years } \\
\hline$<65$ & $46(82.1)$ & $46(92.0)$ & 0.160 & $20(80.0)$ & $26(83.9)$ & 0.738 \\
\hline$\geq 65$ & $10(17.9)$ & $4(8.0)$ & & $5(20.0)$ & $5(16.1)$ & \\
\hline \multicolumn{7}{|l|}{ Sex } \\
\hline Male & $34(60.7)$ & $29(58.0)$ & 0.844 & $11(44.0)$ & $23(74.2)$ & 0.029 \\
\hline Female & $22(39.3)$ & $21(42.0)$ & & $14(56.0)$ & $8(25.8)$ & \\
\hline \multicolumn{7}{|l|}{ Race } \\
\hline White & $55(98.2)$ & $12(24.0)$ & $<0.001$ & $24(96.0)$ & $31(100.0)$ & 0.446 \\
\hline Black & $1(1.8)$ & $1(2.0)$ & & $1(4.0)$ & 0 & \\
\hline Asian & & $37(74.0)$ & & - & - & \\
\hline \multicolumn{7}{|l|}{ ECOG PS } \\
\hline 0 & $36(64.3)$ & $33(66.0)$ & 1.000 & $14(56.0)$ & $22(71.0)$ & 0.256 \\
\hline 1 & 19 (33.9) & $17(34.0)$ & & $11(44.0)$ & $8(25.8)$ & \\
\hline Not reported & $1(1.8)$ & - & & - & $1(3.2)$ & \\
\hline
\end{tabular}

Table 3. Summary of trends for objective response rate comparison between cohorts within SNP subgroups.

\begin{tabular}{|c|c|c|c|}
\hline SNP subgroup & Treatment-naive & Previously treated & Combined \\
\hline \multicolumn{4}{|c|}{ VEGFR2 rs7692791, T/T genotype } \\
\hline $\mathrm{n}$ & 7 & 6 & 13 \\
\hline ORR $(95 \% \mathrm{Cl})$ & $14.3(0.4-57.9)$ & $66.7(22.3-95.7)$ & $38.5(13.9-68.4)$ \\
\hline OR $(95 \% \mathrm{Cl})$ & $0.08(0.0-1.9)$ & - & - \\
\hline $\mathrm{p}$-value & $0.103^{\dagger}$ & - & - \\
\hline \multicolumn{4}{|c|}{ VEGFA rs2010963, G/G genotype } \\
\hline ORR $(95 \% \mathrm{Cl})$ & $0.0(0.0-28.5)$ & $38.5(13.9-68.4)$ & $20.8(7.1-42.2)$ \\
\hline OR $(95 \% \mathrm{Cl})$ & 0.00 & - & - \\
\hline$p$-value & $0.041^{\dagger}$ & - & - \\
\hline \multicolumn{4}{|c|}{ VEGFA rs833068, G/G genotype } \\
\hline $\mathrm{n}$ & 11 & 13 & 24 \\
\hline $\mathrm{n}$ & 10 & 13 & 23 \\
\hline ORR $(95 \% \mathrm{Cl})$ & $0.0(0.0-30.8)$ & $38.5(13.9-68.4)$ & $21.7(7.5-43.7)$ \\
\hline OR $(95 \% \mathrm{Cl})$ & 0.00 & - & - \\
\hline $\mathrm{p}$-value & $0.046^{\dagger}$ & - & - \\
\hline
\end{tabular}

Comparison between cohorts within each SNP subgroup

There were no significant associations between genotype and PFS or OS (Supplementary Tables 3 \& 4 ). There were no statistically significant associations between genotype and ORR after Bonferroni adjustment (Supplementary Table 5). However, the following trends were reported (Table 3): higher ORR in the previously treated cohort versus the treatment-naive cohort for the homozygous genotype G/G of VEGFA rs2010963 (38.5 vs 0.0\%; $\mathrm{p}=0.041$ ); 


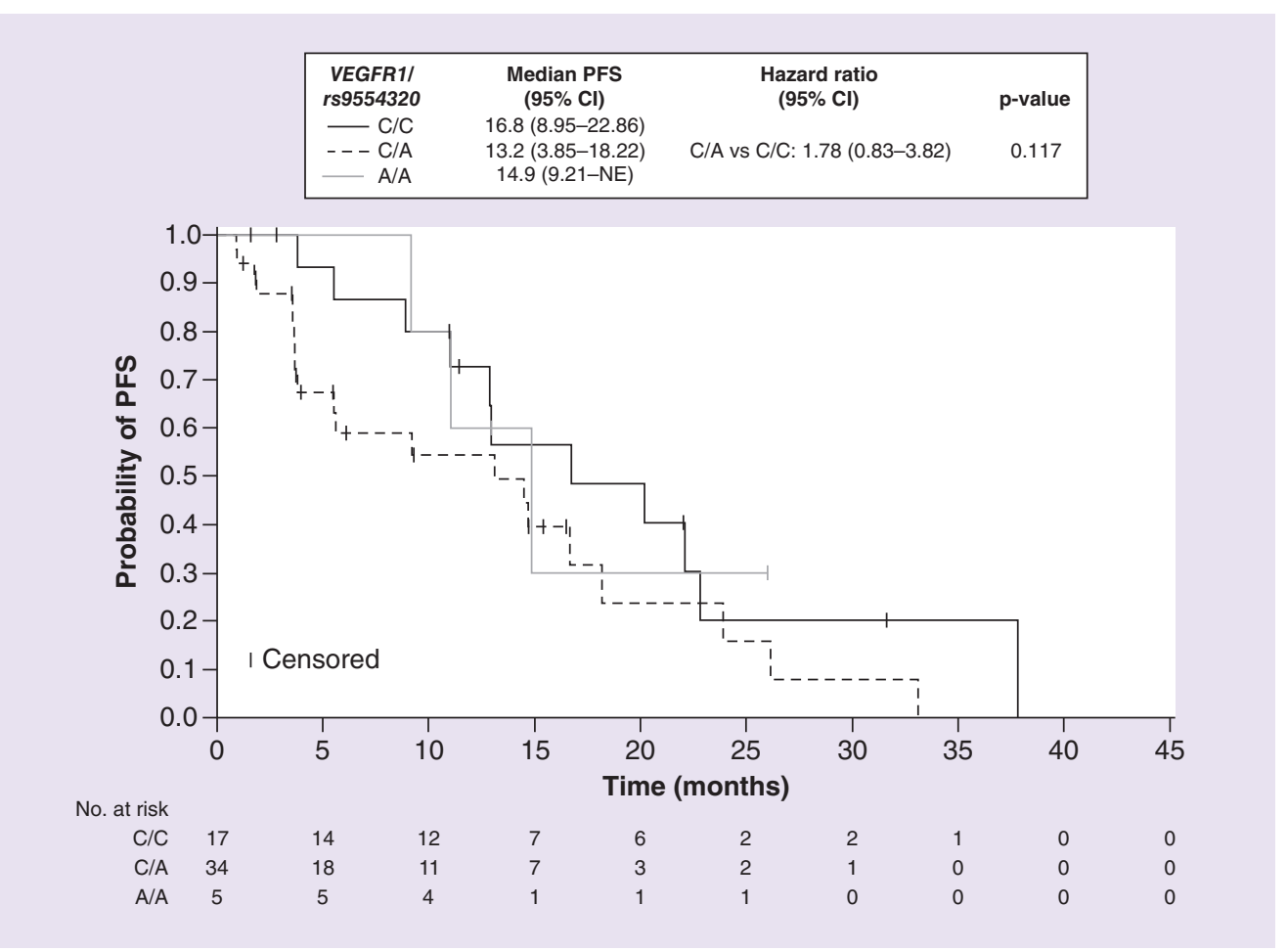

Figure 1. Kaplan-Meier plot of progression-free survival by VEGFR1 rs9554320 genotype in the combined cohort. NE: Non estimable; PFS: Progression-free survival.

the homozygous genotype $\mathrm{G} / \mathrm{G}$ of $V E G F A$ rs 833068 (38.5 vs $0.0 \%$; $\mathrm{p}=0.041$ ); and the heterozygous genotype $\mathrm{A} / \mathrm{C}$ of VEGFR1 rs9582036 (38.5 vs $0.0 \% ; \mathrm{p}=0.046$ ). Additionally, the homozygous genotype T/T of VEGFR2 rs 7692791 showed a trend toward a lower ORR in the treatment-naive cohort compared with the previously treated cohort (14.3 vs 66.7\%; odds ratio [OR]: 0.08; 95\% CI: $0.0-1.9 ; \mathrm{p}=0.103$ ).

\section{Comparison of SNP genotype subgroups within each cohort \& combined}

There were no significant associations between genotype and PFS or OS, regardless of prior line of treatment (Supplementary Tables 6 \& 7). However, there was a trend toward shorter PFS in patients with VEGFR1 rs9554320 $\mathrm{C} / \mathrm{A}$ versus C/C (hazard ratio: 1.78; 95\% CI: 0.83-3.82; $\mathrm{p}=0.117$; Figure 1). Additionally, shorter PFS was also observed in patients with VEGFR1 rs9582036 A/C versus A/A (hazard ratio: 1.88; 95\% CI: 0.9-3.93; $\mathrm{p}=0.102$; Figure 2). Some patients ( $\mathrm{n}=31$ ) were still in follow-up at the time of the analyses cut-off date (19 March 2016).

The heterozygous genotype G/A of IL1B rs 16944 was significantly associated with a higher ORR versus the homozygous genotype G/G (46.4 vs 4.5\%; OR: 18.2; 95\% CI: 2.2-809.3; p = 0.001; Table 4). In addition, in the previously treated cohort, there was a trend toward lower ORR in patients with the heterozygous genotype $\mathrm{T} / \mathrm{C}$ of VEGFR2 rs 7692791 compared with patients with the homozygous genotype T/T (16.7 vs 66.7\%; OR: 0.10; $95 \%$ CI: $0.0-1.2 ; \mathrm{p}=0.038)$. In the combined cohort, this trend was also observed (16.7 vs 38.5\%; OR: $0.32 ; 95 \%$ CI: $0.1-1.8 ; \mathrm{p}=0.140$; Table 4). No other associations between ORR and genotype were reported (Supplementary Table 8).

\section{Discussion}

In these exploratory analyses, after adjustment for multiplicity, no statistically significant correlations were observed between the VEGF pathway SNPs investigated and PFS or OS; however G/A of IL1B rs 16944 was significantly associated with a higher ORR than the homozygous genotype G/G. Although not statistically significant, a trend toward shorter PFS in patients with VEGFR1 rs $9554320 \mathrm{C} / \mathrm{A}$ versus $\mathrm{C} / \mathrm{C}$ and VEGFR1 rs9582036 A/C versus A/A was observed. A trend toward a lower ORR was also noted in patients with VEGFR2 rs $7692791 \mathrm{~T} / \mathrm{C}$ versus $\mathrm{T} / \mathrm{T}$. 


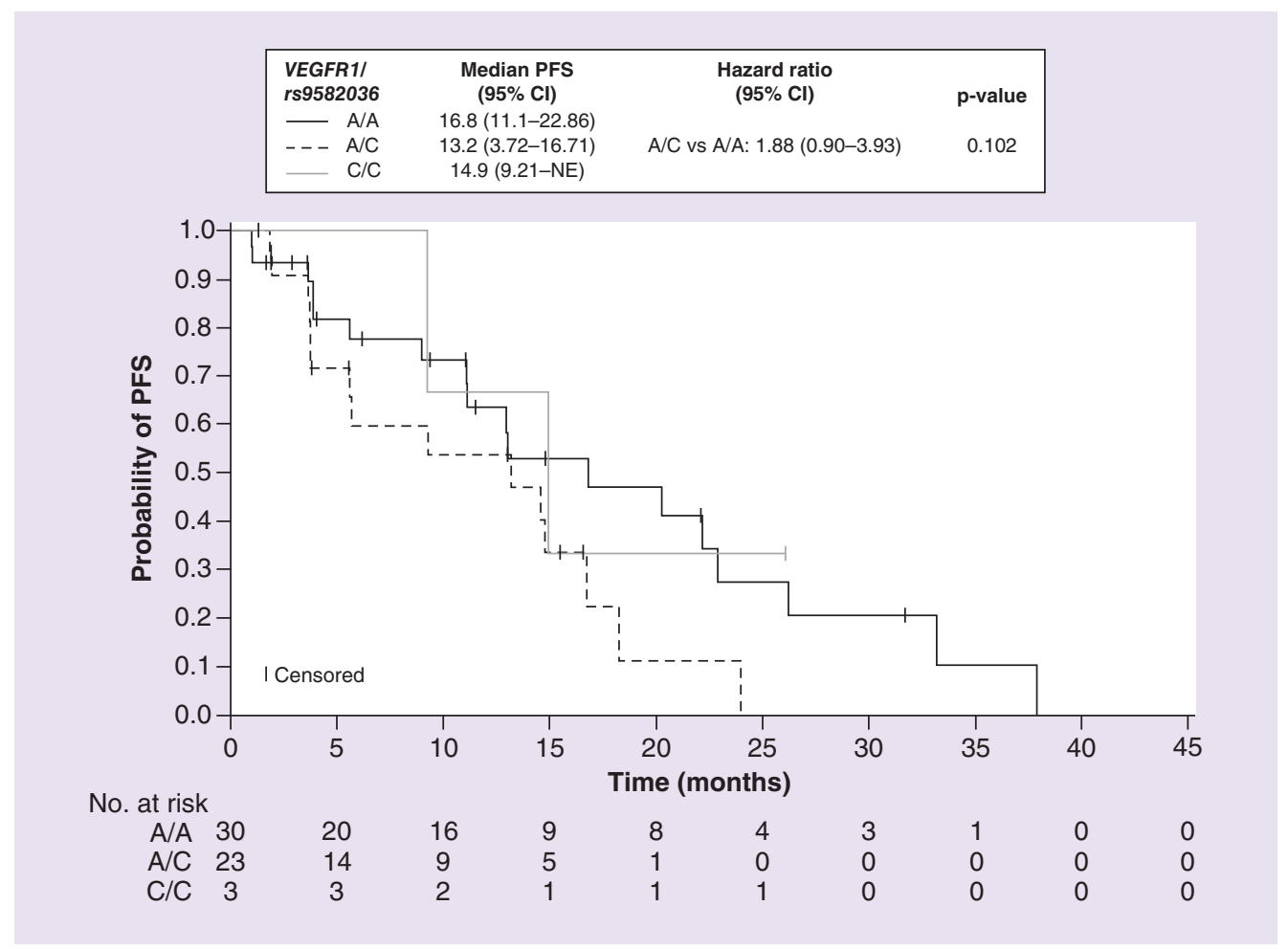

Figure 2. Kaplan-Meier plot of progression-free survival by VEGFR1 rs9582036 genotype in the combined cohort. NE: Non estimable; PFS: Progression-free survival.

\begin{tabular}{|c|c|c|c|c|c|c|c|c|c|}
\hline \multirow{2}{*}{$\begin{array}{l}\text { SNP genotype } \\
\text { subgroup } \\
\text { IL1B rs } 16944\end{array}$} & \multicolumn{3}{|c|}{ Treatment-naive } & \multicolumn{3}{|c|}{ Previously treated } & \multicolumn{3}{|c|}{ Combined } \\
\hline & $\begin{array}{l}\mathrm{G} / \mathrm{G} \\
\mathrm{n}=13\end{array}$ & $\begin{array}{l}G / A \\
n=9\end{array}$ & $\begin{array}{l}A / A \\
n=3\end{array}$ & $\begin{array}{l}G / G \\
n=9\end{array}$ & $\begin{array}{l}\mathrm{G} / \mathrm{A} \\
\mathrm{n}=19\end{array}$ & $\begin{array}{l}A / A \\
n=3\end{array}$ & $\begin{array}{l}\mathrm{G} / \mathrm{G} \\
\mathrm{n}=22\end{array}$ & $\begin{array}{l}\mathrm{G} / \mathrm{A} \\
\mathrm{n}=28\end{array}$ & $\begin{array}{l}A / A \\
n=6\end{array}$ \\
\hline $\begin{array}{l}\text { ORR } \\
(95 \% \mathrm{Cl})\end{array}$ & $\begin{array}{l}7.7 \\
(0.2-36.0)\end{array}$ & $\begin{array}{l}33.3 \\
(7.5-70.1)\end{array}$ & $\begin{array}{l}33.3 \\
(0.8-90.6)\end{array}$ & $\begin{array}{l}0.0 \\
(0.0-33.6)\end{array}$ & $\begin{array}{l}52.6 \\
(28.9-75.6)\end{array}$ & $\begin{array}{l}0.0 \\
(0.0-70.8)\end{array}$ & $\begin{array}{l}4.5 \\
(0.1-22.8)\end{array}$ & $\begin{array}{l}46.4 \\
(27.5-66.1)\end{array}$ & $\begin{array}{l}16.7 \\
(0.4-64.1)\end{array}$ \\
\hline $\begin{array}{l}\text { OR } \\
(95 \% \mathrm{Cl})\end{array}$ & - & $\begin{array}{l}6.00 \\
(0.4-340.6)\end{array}$ & $\begin{array}{l}6.00 \\
(0.1-509.0)\end{array}$ & - & - & - & - & $\begin{array}{l}18.2 \\
(2.2-809.3)\end{array}$ & $\begin{array}{l}4.20 \\
(0.0-344.9)\end{array}$ \\
\hline$p$-value & - & $0.264^{\dagger}$ & $0.350^{\dagger}$ & - & $0.010^{\dagger}$ & - & - & $0.001^{\dagger}$ & $0.389^{\dagger}$ \\
\hline $\begin{array}{l}\text { VEGFR2 } \\
\text { rs7692791 }\end{array}$ & $\begin{array}{l}T / T \\
n=7\end{array}$ & $\begin{array}{l}\mathrm{T} / \mathrm{C} \\
\mathrm{n}=12\end{array}$ & $\begin{array}{l}C / C \\
n=6\end{array}$ & $\begin{array}{l}T / T \\
n=6\end{array}$ & $\begin{array}{l}\mathrm{T} / \mathrm{C} \\
\mathrm{n}=18\end{array}$ & $\begin{array}{l}C / C \\
n=7\end{array}$ & $\begin{array}{l}\mathrm{T} / \mathrm{T} \\
\mathrm{n}=13\end{array}$ & $\begin{array}{l}T / C \\
n=30\end{array}$ & $\begin{array}{l}C / C \\
n=13\end{array}$ \\
\hline $\begin{array}{l}\text { ORR } \\
(95 \% \mathrm{Cl})\end{array}$ & $\begin{array}{l}14.3 \\
(0.4-57.9)\end{array}$ & $\begin{array}{l}16.7 \\
(2.1-48.4)\end{array}$ & $\begin{array}{l}33.3 \\
(4.3-77.7)\end{array}$ & $\begin{array}{l}66.7 \\
(22.3-95.7)\end{array}$ & $\begin{array}{l}16.7 \\
(3.6-41.4)\end{array}$ & $\begin{array}{l}42.9 \\
(9.9-81.6)\end{array}$ & $\begin{array}{l}38.5 \\
(13.9-68.4)\end{array}$ & $\begin{array}{l}16.7 \\
(5.6-34.7)\end{array}$ & $\begin{array}{l}38.5 \\
(13.9-68.4)\end{array}$ \\
\hline $\begin{array}{l}\text { OR } \\
(95 \% \mathrm{Cl})\end{array}$ & - & $\begin{array}{l}1.20 \\
(0.1-82.4)\end{array}$ & $\begin{array}{l}3.00 \\
(0.1-205.9)\end{array}$ & - & $\begin{array}{l}0.10 \\
(0.0-1.2)\end{array}$ & $\begin{array}{l}0.38 \\
(0.0-5.5)\end{array}$ & - & $\begin{array}{l}0.32 \\
(0.1-1.8)\end{array}$ & $\begin{array}{l}1.00 \\
(0.2-6.4)\end{array}$ \\
\hline$p$-value & - & $1.000^{\dagger}$ & $0.559^{\dagger}$ & - & $0.038^{\dagger}$ & $0.592^{\dagger}$ & - & $0.140^{\dagger}$ & $1.000^{\dagger}$ \\
\hline
\end{tabular}

Our results support that SNPs of VEGFR may play a role in clinical response to therapy in neuroendocrine tumors. In other tumor types, SNPs in VEGFR1 (VEGFR1 rs9582036 and rs9554320) have been associated with clinical outcomes (ORR, PFS and/or OS) in pancreatic cancer or metastatic colorectal cancer during treatment with bevacizumab, or during treatment with sunitinib in patients with metastatic renal cell carcinoma (RCC) or in the adjuvant RCC setting [18,20-24]. Despite conflicting reports, certain SNPs of VEGFR1 appear to have an impact on clinical response across various tumor types, including in patients with panNETs. In the current analyses, there was a trend for higher ORR in previously treated patients compared with treatment-naive patients 
for certain VEGFA, VEGFR1 and VEGFR2 genotypes. In the adjuvant RCC setting, longer disease-free survival was reported for sunitinib versus placebo in patients with homozygous genotypes C/C for VEGFR1 rs9554320 and $\mathrm{T} / \mathrm{T}$ for VEGFR2 rs2071559 [24]. Although the study was not designed to identify associations between treatment groups, marginally significant interactions between two sunitinib treatment arms and VEGFR3 rs 448012 were seen for clinical outcomes in the RENAL EFFECT study in metastatic RCC [25]. Although no comparisons were made among different treatment groups, SNPs in the VEGFA, VEGFR1, VEGFR2 and/or VEGFR3 genes have been associated with varying clinical outcomes in other trials evaluating sunitinib-treated patients with metastatic RCC [17,18,20,23,26-29], as well as in bevacizumab-treated patients with metastatic colorectal or pancreatic cancer [18,21,22]. These data suggest that further evaluation of the effects of SNPs within VEGFA, VEGFR1, VEGFR2 and VEGFR3 on clinical outcomes across different treatment arms in patients with panNETs is warranted.

Establishing the predictive value of VEGFR SNPs could have important clinical implications. For example, in patients where it is necessary to achieve rapid regression of the tumor, due to pain or other symptoms, it may be possible to differentiate treatment. With relevance to this study, eligible patients with panNETs could be treated with sunitinib instead of chemotherapy, thus improving clinical outcomes and avoiding the toxicity associated with chemotherapy. Indeed, the ultimate goal of identifying SNPs associated with clinical outcome is to provide individualized treatment regimens that will optimize the effectiveness and safety of available treatments; however, several obstacles remain before SNP analysis can be routinely used in clinical practice. The majority of SNP studies are hypothesis generating and require validation in larger prospective studies. Furthermore, many SNP studies rely on PCR-based amplification of DNA and subsequent allelic discrimination assays. While minimally invasive, effective and relatively inexpensive, this method requires many steps and is of a low-moderate throughput [30]. The development of cost-effective, higher throughput diagnostic techniques would greatly aid the implementation of routine SNP analysis to the clinic.

Our analyses did not investigate VEGFR3 SNPs, reported by others to be correlated with a decreased tumor response to pazopanib [31], divergent tumor responses to sunitinib [18,20,25,32-34] and poor prognosis in patients with gastroenteropancreatic neuroendocrine neoplasms [35]. A recent Spanish multicenter SNP analysis in patients with well-differentiated panNETs reported that VEGFR3 rs307826 and rs307821 predicted lower OS, but not PFS [34]; however, following correction for multiple testing, no correlations remained significant. Consistent with our study, no statistically significant correlation was observed between PFS or OS and the SNPs VEGFA rs2010963, ABCB1 rs1128503 or $A B C B 1$ rs2032582 [34].

$I L 1 B$ has been shown to be involved in panNET etiology and development [19,36]. Analysis of SNPs associated with tumor development may prove to be important in the diagnosis and identification of high-risk individuals. Polymorphisms in the displacement loop region of mitochondrial DNA have been identified that may be implicated in gastroenteropancreatic neuroendocrine neoplasms development [37] and survival [38]. Others have reported that polymorphisms in $C D K N 2 A / B$ are risk factors for panNET development [39]. IL1B SNPs have been reported to be associated with the development of prostate cancer [40], but have no influence on outcomes in non-small-cell lung cancer [41]. However, this is the first report of IL1B SNPs leading to differences in treatment response in patients with panNETs. Therefore, specific further prospective investigation in patients with panNETs is warranted on this target to confirm the correlation with sunitinib treatment.

Our study adds to a growing body of evidence that SNPs in the VEGFR signalling pathway may help predict clinical responses in patients receiving sunitinib. Due to differences between genotyped and nongenotyped patients, the population used in this genotyping analysis could not be representative of the overall study population; thus, conclusions derived from the analysis of the genotyped population should not be extrapolated to the full study population. Due to the rare nature of panNETs, it is challenging to recruit large numbers of patients for clinical trials and subsequent analyses. The genotyped population $(n=56)$ of our study was of a comparable size with other SNP studies in patients with panNETs, including studies by Jiménez-Fonseca et al. $(\mathrm{n}=43)$ [34], Karakaxas et al. $(\mathrm{n}=51)$ [36] and Cigrovski Berković et al. $(\mathrm{n}=60)$ [19]. Consequently, our analyses, like many SNP panNET studies, are also limited by the small sample sizes in some of the subgroups, as well as the exploratory nature of the study. Therefore, these findings will need to be validated in larger prospective studies in patients with panNETs.

\section{Conclusion}

No statistically significant correlations were observed between the VEGF pathway SNPs investigated and PFS or OS. A significant association was seen for IL1B rs 16944 and ORR, which is consistent with the role of ILIB in panNET etiology and development. Some associations were observed between ORR and SNPs in VEGFA, VEGFR1 
and VEGFR2 genotypes, although most correlations were not significant after adjustment for multiplicity. Further investigations in homogeneous, prospective studies are needed to elucidate the role of these SNPs in the treatment of patients with locally advanced unresectable/metastatic well-differentiated panNETs.

\section{Summary points}

- Well-differentiated pancreatic neuroendocrine tumors (panNETs) are highly angiogenic tumors whose incidence appears to be increasing.

- Sunitinib inhibits angiogenic activity through the VEGF pathway and has demonstrated improvements in progression-free survival (PFS) in patients with well-differentiated panNETs.

- Variability in the clinical response to sunitinib may be due to single-nucleotide polymorphisms in sunitinib targets.

- This study investigated potential associations between clinical outcomes and 12 SNPs in a subset of patients from a sunitinib Phase IV trial in patients with panNETs.

- Fifty-six (25 treatment-naive and 31 previously treated) patients consented and were genotyped.

- There were no significant associations between genotype and PFS or overall survival, but there was a trend toward shorter PFS (hazard ratio [95\% CI]) in patients with VEGFR1 rs9554320 C/A versus C/C (1.78 [0.83-3.82]; $\mathrm{p}=0.117)$ and VEGFR1 rs9582036 A/C versus A/A (1.88 [0.9-3.93]; $\mathrm{p}=0.102)$.

- Correlations between overall response rate and VEGFA rs2010963 and rs833068, VEGFR1 rs9582036 and VEGFR2 rs7692791 were observed, although they were not statistically significant.

- Higher ORR was significantly associated with IL1B rs16944 G/A versus G/G (46.4 vs 4.5\%; $p=0.001)$ in the combined group.

- This is the first report of IL1B SNPs leading to differentiated treatment responses in patients with panNETs.

- Further study is warranted to confirm the association of IL1B and VEGFR1 SNPs with treatment response. These findings may have implications for future treatment decision making.

Supplementary data

To view the supplementary data that accompany this paper please visit the journal website at: www.futuremedicine.com/doi/sup $\mathrm{pl} / 10.2217 /$ fon-2018-0934

Author contributions

All authors were involved in the study conception/design, or the acquisition, analysis or interpretation of data. All authors contributed to the drafting of the manuscript and approved the final version. All authors contributed equally to the creation of this manuscript.

\section{Acknowledgments}

The authors thank patients in this Phase IV trial who provided blood samples for analytical purposes and trial coordinators and local sub-investigators for their contribution to the study. They also acknowledge the contribution of the following individuals at Pfizer: L Wood and J-C Marshall for their assistance with laboratory study execution and review of the article, and C Bolanos and L Harty for biospecimens reconciliation and informed consent review.

\section{Financial \& competing interests disclosure}

This study was sponsored by Pfizer. N Fazio has received honoraria from Pfizer; he has participated in advisory boards for Novartis, Pfizer and Ipsen, Merck Serono, MSD and AAA. His institution has also received research funds from Novartis and Merck Serono. AE Croitoru has received honoraria from Amgen, Merck-Serono and Roche; has received research funding from Merck Serono, MSD, Roche and Pfizer; has participated in advisory boards for Amgen, Sanofi, Merck Serono, Roche and Eli Lilly; has received lecture fees from Pfizer; and has received travel support from Amgen, Sanofi, Merck Serono and Roche. M Schenker has received clinical trial grants from Bristol-Myers Squibb, Pfizer, Novartis, Amgen, Roche, Eli Lilly, AbbVie, Synta Pharmaceuticals, Astellas, Boehringer Ingelheim, Bayer, MSD, Merck Serono, Gilead and Daichii Sankyo. His institution, Centrul de Oncologie Sf Nectarie, Craiova, has also received clinical trials grants from the same companies. J-F Martini, S Li, B Rosbrook and K Fernandez are employees of Pfizer. J Tomasek is a consultant for Bristol-Myers Squibb, Pfizer and Bayer; has received honoraria from Bristol-Myers Squibb, Pfizer, Amgen, Merck, Roche and Bayer; and has received travel support from Ipsen, Pfizer, Amgen, Merck, Roche and Bayer. E ThiisEvensen has received research funding from Novartis and Ipsen; and speaker honorarium from Novartis, Ipsen, Pfizer and MSD. $\mathrm{MH}$ Kulke has participated in advisory boards for Ipsen and Novartis. E Raymond is a consultant for and has received grants for 
clinical trials from Ipsen, Novartis, Eli Lilly and Pfizer. The authors have no other relevant affiliations or financial involvement with any organization or entity with a financial interest in or financial conflict with the subject matter or materials discussed in the manuscript apart from those disclosed.

Medical writing support was provided by AM McGonigal and L Adams of Engage Scientific Solutions, and funded by Pfizer.

\section{Data sharing statement}

The authors certify that this manuscript reports the secondary analysis of clinical trial data that have been shared with them, and that the use of this shared data is in accordance with the terms (if any) agreed upon their receipt. The source of this data is: ClinicalTrials.gov, identifier NCT01525550.

Upon request, and subject to certain criteria, conditions and exceptions (see www.pfizer.com/science/clinical-trials/trial-data-an d-results for more information), Pfizer will provide access to individual de-identified participant data from Pfizer-sponsored global interventional clinical studies conducted for medicines, vaccines and medical devices for indications that have been approved in the USA and/or EU; or in programs that have been terminated (i.e., development for all indications has been discontinued). Pfizer will also consider requests for the protocol, data dictionary and statistical analysis plan. Data may be requested from Pfizer trials 24 months after study completion. The de-identified participant data will be made available to researchers whose proposals meet the research criteria and other conditions, and for which an exception does not apply, via a secure portal. To gain access, data requestors must enter into a data access agreement with Pfizer.

\section{Ethical conduct of research}

The authors state that they have obtained appropriate institutional review board approval or have followed the principles outlined in the Declaration of Helsinki for all human or animal experimental investigations. For investigations involving human subjects, informed consent has been obtained from the participants involved.

\section{Open access}

This work is licensed under the Attribution-NonCommercial-NoDerivatives 4.0 Unported License. To view a copy of this license, visit http://creativecommons.org/licenses/by-nc-nd/4.0/

\section{Data disposition}

The trial is registered on ClinicalTrials.gov, identifier NCT01525550.

\section{References}

Papers of special note have been highlighted as: $\bullet$ of interest

1. Halfdanarson TR, Rabe KG, Rubin J, Petersen GM. Pancreatic neuroendocrine tumors (PNETs): incidence, prognosis and recent trend toward improved survival. Ann. Oncol. 19(10), 1727-1733 (2008).

2. McKenna LR, Edil BH. Update on pancreatic neuroendocrine tumors. Gland Surg. 3(4), 258-275 (2014).

3. Sharma J, Duque M, Saif MW. Emerging therapies and latest development in the treatment of unresectable pancreatic neuroendocrine tumors: an update for clinicians. Therap. Adv. Gastroenterol. 6(6), 474-490 (2013).

4. Zhang J, Jia Z, Li Q et al. Elevated expression of vascular endothelial growth factor correlates with increased angiogenesis and decreased progression-free survival among patients with low-grade neuroendocrine tumors. Cancer 109(8), 1478-1486 (2007).

5. Raymond E, Dahan L, Raoul JL et al. Sunitinib malate for the treatment of pancreatic neuroendocrine tumors. N. Engl. J. Med. 364(6), 501-513 (2011).

6. Raymond E, Kulke MH, Qin S et al. Efficacy and safety of sunitinib in patients with well-differentiated pancreatic neuroendocrine tumours. Neuroendocrinology 107(3), 237-245 (2018).

- The Phase IV study from which the dataset used for this analysis was obtained.

7. Oberg K, Knigge U, Kwekkeboom D, Perren A, Group EGW. Neuroendocrine gastro-entero-pancreatic tumors: ESMO Clinical Practice Guidelines for diagnosis, treatment and follow-up. Ann. Oncol. 23(Suppl. 7), vii124-130 (2012).

- European Society for Medical Oncology clinical practice guidelines that describe the recommended use of sunitinib in patients with pancreatic neuroendocrine tumors (panNETs).

8. Pusceddu S, Buzzoni R, De Braud F. Pancreatic well-differentiated neuroendocrine neoplasms (pWDNENs): what place for everolimus and sunitinib derived from ESMO clinical practice guidelines in the therapeutic algorithm? Ann. Oncol. 24(5), 1415-1416 (2013).

9. Mizuno Y, Kudo A, Akashi T et al. Sunitinib shrinks NET-G3 pancreatic neuroendocrine neoplasms. J. Cancer Res. Clin. Oncol. 144(6), 1155-1163 (2018).

10. Rinzivillo M, Fazio N, Pusceddu $S$ et al. Sunitinib in patients with pre-treated pancreatic neuroendocrine tumors: a real-world study. Pancreatology 18(2), 198-203 (2018). 
11. Valle JW, Borbath I, Rosbrook B, Fernandez K, Raymond E. Sunitinib in patients with pancreatic neuroendocrine tumors: update of safety data. Future Oncol. 15(11), 1219-1230 (2019).

- Long-term evaluation of the sunitinib safety profile in patients with well-differentiated panNETs.

12. Porta C, Gore ME, Rini BI et al. Long-term safety of sunitinib in metastatic renal cell carcinoma. Eur. Urol. 69(2), 345-351 (2016).

13. Erichsen HC, Chanock SJ. SNPs in cancer research and treatment. Br. J. Cancer 90(4), 747-751 (2004).

14. Rodriguez-Antona C, Taron M. Pharmacogenomic biomarkers for personalized cancer treatment. J. Intern. Med. 277(2), 201-217 (2015).

15. Beuselinck B, Lambrechts D, Van Brussel $\mathrm{T}$ et al. Efflux pump ABCB1 single nucleotide polymorphisms and dose reductions in patients with metastatic renal cell carcinoma treated with sunitinib. Acta Oncol. 53(10), 1413-1422 (2014).

16. Diekstra $\mathrm{MH}$, Swen JJ, Boven $\mathrm{E}$ et al. CYP3A5 and $\mathrm{ABCB} 1$ polymorphisms as predictors for sunitinib outcome in metastatic renal cell carcinoma. Eur. Urol. 68(4), 621-629 (2015).

17. Maeng $\mathrm{CH}$, Yi JH, Lee J et al. Effects of single nucleotide polymorphisms on treatment outcomes and toxicity in patients treated with sunitinib. Anticancer Res. 33(10), 4619-4626 (2013).

18. Beuselinck B, Karadimou A, Lambrechts D et al. VEGFR1 single nucleotide polymorphisms associated with outcome in patients with metastatic renal cell carcinoma treated with sunitinib - a multicentric retrospective analysis. Acta Oncol. 53(1), 103-112 (2014).

- Previous multicenter study that identified VEGFR1 single-nucleotide polymorphisms associated with clinical outcomes in patients with renal cell carcinoma.

19. Cigrovski Berković M, Catela Ivković T, Marout J, Zjačić-Rotkvić V, Kapitanović S. Interleukin $1 \beta$ gene single-nucleotide polymorphisms and susceptibility to pancreatic neuroendocrine tumors. DNA Cell Biol. 31(4), 531-536 (2012).

- Previous study that identifies $I L 1 B$ SNPs involved in panNET development and susceptibility.

20. Dornbusch J, Walter M, Gottschalk A et al. Evaluation of polymorphisms in angiogenesis-related genes as predictive and prognostic markers for sunitinib-treated metastatic renal cell carcinoma patients. J. Cancer Res. Clin. Oncol. 142(6), 1171-1182 (2016).

21. Hansen TF, Christensen R, Andersen RF, Garm Spindler KL, Johnsson A, Jakobsen A. The predictive value of single nucleotide polymorphisms in the VEGF system to the efficacy of first-line treatment with bevacizumab plus chemotherapy in patients with metastatic colorectal cancer: results from the Nordic ACT trial. Int. J. Colorectal Dis. 27(6), 715-720 (2012).

22. Lambrechts D, Claes B, Delmar P et al. VEGF pathway genetic variants as biomarkers of treatment outcome with bevacizumab: an analysis of data from the AViTA and AVOREN randomised trials. Lancet Oncol. 13(7), 724-733 (2012).

23. Diekstra MH, Swen JJ, Gelderblom H, Guchelaar HJ. A decade of pharmacogenomics research on tyrosine kinase inhibitors in metastatic renal cell cancer: a systematic review. Expert Rev. Mol. Diagn. 16(5), 605-618 (2016).

24. George DJ, Martini JF, Staehler M et al. Phase III trial of adjuvant sunitinib in patients with high-risk renal cell carcinoma: exploratory pharmacogenomic analysis. Clin. Cancer Res. 24(5), 1165-1173 (2019).

25. Motzer RJ, Figlin RA, Martini JF et al. Germline genetic biomarkers of sunitinib efficacy in advanced renal cell carcinoma: results from the RENAL EFFECT trial. Clin. Genitourin. Cancer 15(5), 526-533 (2017).

26. Beuselinck B, Jean-Baptiste J, Schöffski P et al. Validation of VEGFR1 rs9582036 as predictive biomarker in metastatic clear-cell renal cell carcinoma patients treated with sunitinib. BJU Int. 118(6), 890-901 (2016).

27. Scartozzi M, Bianconi M, Faloppi L et al. VEGF and VEGFR polymorphisms affect clinical outcome in advanced renal cell carcinoma patients receiving first-line sunitinib. Br. J. Cancer 108(5), 1126-1132 (2013).

28. Bianconi M, Faloppi L, Loretelli $\mathrm{C}$ et al. Angiogenesis genotyping in the selection of first-line treatment with either sunitinib or pazopanib for advanced renal cell carcinoma. Oncotarget 7(25), 37599-37607 (2016).

29. Liu R, Wang X, Li W et al. Influence of VEGFR single nucleotide polymorphisms on the efficacy of sunitinib therapy against renal cell carcinoma. Oncol. Lett. 13(1), 201-205 (2017).

30. Maring ED, Tawadros PS, Steer CJ, Lee JT. Systematic review of candidate single-nucleotide polymorphisms as biomarkers for responsiveness to neoadjuvant chemoradiation for rectal cancer. Anticancer Res. 35(7), 3761-3766 (2015).

31. Grande E, Capdevila J, Castellano D et al. Pazopanib in pretreated advanced neuroendocrine tumors: a Phase II, open-label trial of the Spanish Task Force Group for Neuroendocrine Tumors (GETNE). Ann Oncol. 26(9), 1987-1993 (2015).

32. Garcia-Donas J, Esteban E, Leandro-Garcia LJ et al. Single nucleotide polymorphism associations with response and toxic effects in patients with advanced renal-cell carcinoma treated with first-line sunitinib: a multicentre, observational, prospective study. Lancet Oncol. 12(12), 1143-1150 (2011).

33. Miao C, Cao J, Wang Y, Liu B, Wang Z. Effects of VEGF and VEGFR polymorphisms on the outcome of patients with metastatic renal cell carcinoma treated with sunitinib: a systematic review and meta-analysis. Oncotarget 8(40), 68854-68862 (2017).

34. Jimenez-Fonseca P, Martin MN, Carmona-Bayonas A et al. Biomarkers and polymorphisms in pancreatic neuroendocrine tumors treated with sunitinib. Oncotarget 9(97), 36894-36905 (2018).

- Previous study that describes the potential association of VEGFR-3 SNPs and overall survival in sunitinib-treated patients with well-differentiated panNETs. 
35. Berardi R, Torniai M, Partelli $S$ et al. Impact of vascular endothelial growth factor (VEGF) and vascular endothelial growth factor receptor (VEGFR) single nucleotide polymorphisms on outcome in gastroenteropancreatic neuroendocrine neoplasms. PLoS ONE 13(5), e0197035 (2018).

36. Karakaxas D, Sioziou A, Aravantinos G et al. Genetic polymorphisms of interleukin $1 \beta$ gene and sporadic pancreatic neuroendocrine tumors susceptibility. World J. Gastrointest. Oncol. 8(6), 520-525 (2016).

37. Er LM, Wu ML, Gao Y, Wang SJ, Li Y. Identification of sequence polymorphisms in the displacement loop region of mitochondrial DNA as a risk factor for gastroenteropancreatic neuroendocrine neoplasm. J. Clin. Lab. Anal. 31(5), doi:10.1002/jcla.22078 (2017).

38. Er LM, Li Y, Er L et al. Associations between single nucleotide polymorphisms in the mitochondrial DNA D-loop region and outcome of gastroenteropancreatic neuroendocrine neoplasm. Ann. Clin. Lab. Sci. 48(3), 333-337 (2018).

39. Campa D, Capurso G, Pastore $M$ et al. Common germline variants within the CDKN2A/2B region affect risk of pancreatic neuroendocrine tumors. Sci. Rep. 6, 39565 (2016).

40. Yencilek F, Yildirim A, Yilmaz SG et al. Investigation of interleukin-1 $\beta$ polymorphisms in prostate cancer. Anticancer Res. 35(11), 6057-6061 (2015).

41. Perez-Ramirez C, Canadas-Garre M, Alnatsha A et al. Interleukins as new prognostic genetic biomarkers in non-small-cell lung cancer. Surg. Oncol. 26(3), 278-285 (2017). 
